This item was submitted to Loughborough's Research Repository by the author.

Items in Figshare are protected by copyright, with all rights reserved, unless otherwise indicated.

\title{
Deformed Macdonald-Ruijsenaars operators and super Macdonald polynomials
}

\section{PLEASE CITE THE PUBLISHED VERSION}

http://dx.doi.org/10.1007/s00220-009-0779-3

\section{PUBLISHER}

(c) Springer-Verlag

\section{VERSION}

AM (Accepted Manuscript)

\section{PUBLISHER STATEMENT}

This work is made available according to the conditions of the Creative Commons Attribution-NonCommercialNoDerivatives 4.0 International (CC BY-NC-ND 4.0) licence. Full details of this licence are available at: https://creativecommons.org/licenses/by-nc-nd/4.0/

\section{LICENCE}

CC BY-NC-ND 4.0

\section{REPOSITORY RECORD}

Sergeev, A.N., and A.P. Veselov. 2019. "Deformed Macdonald-ruijsenaars Operators and Super Macdonald Polynomials”. figshare. https://hdl.handle.net/2134/16205. 


\title{
DEFORMED MACDONALD-RUIJSENAARS OPERATORS AND SUPER MACDONALD POLYNOMIALS
}

\author{
A.N. SERGEEV AND A.P. VESELOV
}

\begin{abstract}
It is shown that the deformed Macdonald-Ruijsenaars operators can be described as the restrictions on certain affine subvarieties of the usual Macdonald-Ruijsenaars operator in infinite number of variables. The ideals of these varieties are shown to be generated by the Macdonald polynomials related to Young diagrams with special geometry. The super Macdonald polynomials and their shifted version are introduced, the combinatorial formulas for them are given.
\end{abstract}

\section{INTRODUCTION}

In this paper we investigate the properties of the deformed MacdonaldRuijsenaars (MR) operators introduced in [1]

$$
\mathcal{M}_{n, m, q, t}=\frac{1}{1-q} \sum_{i=1}^{n} A_{i}\left(T_{q, x_{i}}-1\right)+\frac{1}{1-t} \sum_{j=1}^{m} B_{j}\left(T_{t, y_{j}}-1\right)
$$

where

$$
A_{i}=\prod_{k \neq i}^{n} \frac{\left(x_{i}-t x_{k}\right)}{\left(x_{i}-x_{k}\right)} \prod_{j=1}^{m} \frac{\left(x_{i}-q y_{j}\right)}{\left(x_{i}-y_{j}\right)}, \quad B_{j}=\prod_{i=1}^{n} \frac{\left(y_{j}-t x_{i}\right)}{\left(y_{j}-x_{i}\right)} \prod_{l \neq j}^{m} \frac{\left(y_{j}-q y_{l}\right)}{\left(y_{j}-y_{l}\right)}
$$

and $T_{q, x_{i}}, T_{t, y_{j}}$ are the "shift" operators:

$$
\begin{aligned}
& \left(T_{q, x_{i}} f\right)\left(x_{1}, \ldots, x_{i}, \ldots, x_{n}, y_{1}, \ldots, y_{m}\right)=f\left(x_{1}, \ldots, q x_{i}, \ldots, x_{n}, y_{1}, \ldots, y_{m}\right) \\
& \left(T_{t, y_{j}} f\right)\left(x_{1}, \ldots, x_{n}, y_{1}, \ldots, y_{j}, \ldots, y_{m}\right)=f\left(x_{1}, \ldots, x_{n}, y_{1}, \ldots, t y_{j}, \ldots, y_{m}\right) .
\end{aligned}
$$

More precisely, we generalise the results of our paper [2] by showing that the deformed MR operator can be described as the restriction of the usual Macdonald-Ruijsenaars operator [3, 4]

$$
\mathcal{M}_{q, t}=\frac{1}{1-q} \sum_{i \geq 1} \prod_{j \neq i} \frac{z_{i}-t z_{j}}{z_{i}-z_{j}}\left(T_{q, z_{i}}-1\right)
$$

for infinite number of variables $z_{i}$ onto certain subvarieties $\Delta_{n, m, q, t}$.

As well as our previous paper [2] this work is based on the theory of Macdonald polynomials [4] and shifted Macdonald polynomials developed by Knop, Sahi and Okounkov [5, 6, 7, 8]. The paper [9] by B. Feigin, Jimbo, Miwa and Mukhin was very useful for us in understanding the role of special parameters in this problem. Another important relevant work is the paper [10] by Chalykh, who used a different technique to derive and investigate 
the deformed MR operator in the case $m=1$, which was the first case when the deformed Calogero-Moser systems were discovered (see [11]).

The structure of the paper is the following. First we review some basic facts from the theory of Macdonald polynomials and Cherednik-Dunkl operators. The main results about deformed MR operators are proved in section 5. We introduce the super Macdonald polynomials as the restriction of the usual Macdonald polynomials on $\Delta_{n, m, q, t}$. In section 6 we define their shifted versions and show that for any shifted super Macdonald polynomial there exists a difference operator commuting with $\mathcal{M}_{n, m, q, t}$ (a deformed version of Harish-Chandra homomorphism). In the last section we present some combinatorial formulas for the super Macdonald polynomials and their shifted versions generalising Okounkov's result [8].

\section{Symmetric FunCtions And MaCdonald POlynomials}

In this section we recall some general facts about symmetric functions and Macdonald polynomials mainly following Macdonald's classical book [4]. It will be convenient for us to use instead of the parameters $q, t$ in Macdonald's notations of Macdonald polynomials the parameters $q, t^{-1}$.

Let $P_{N}=\mathbb{C}\left[x_{1}, \ldots, x_{N}\right]$ be the polynomial algebra in $N$ independent variables and $\Lambda_{N} \subset P_{N}$ be the subalgebra of symmetric polynomials.

A partition is any sequence

$$
\lambda=\left(\lambda_{1}, \lambda_{2}, \ldots, \lambda_{r} \ldots\right)
$$

of nonnegative integers in decreasing order

$$
\lambda_{1} \geq \lambda_{2} \geq \cdots \geq \lambda_{r} \geq \cdots
$$

containing only finitely many nonzero terms. The number of nonzero terms in $\lambda$ is the length of $\lambda$ denoted by $l(\lambda)$. The sum $|\lambda|=\lambda_{1}+\lambda_{2}+\ldots$ is called the weight of $\lambda$. The set of all partitions of weight $N$ is denoted by $\mathcal{P}_{N}$.

On this set there is a natural involution: in the standard diagrammatic representation [4] it corresponds to the transposition (reflection in the main diagonal). The image of a partition $\lambda$ under this involution is called the conjugate of $\lambda$ and denoted by $\lambda^{\prime}$. This involution will play an essential role in our paper.

Partitions can be used to label the bases in the symmetric algebra $\Lambda_{N}$. There are the following two standard bases in $\Lambda_{N}$, which we are going to use: monomial symmetric polynomials $m_{\lambda}, \lambda \in \mathcal{P}_{N}$, which are defined by

$$
m_{\lambda}\left(x_{1}, \ldots, x_{N}\right)=\sum x_{1}^{a_{1}} x_{2}^{a_{2}} \ldots x_{N}^{a_{N}}
$$

summed over all distinct permutations $a$ of $\lambda=\left(\lambda_{1}, \lambda_{2}, \ldots, \lambda_{N}\right)$ and power sums

$$
p_{\lambda}=p_{\lambda_{1}} p_{\lambda_{2}} \ldots p_{\lambda_{N}}
$$

where

$$
p_{k}=x_{1}^{k}+x_{2}^{k}+\cdots+x_{N}^{k} .
$$


It is well-known [4] that each of these sets of functions with $l(\lambda) \leq N$ form a basis in $\Lambda_{N}$.

We will need the following infinite dimensional versions of both $P_{N}$ and $\Lambda_{N}$. Let $M \leq N$ and $\varphi_{N, M}: P_{N} \longrightarrow P_{M}$ be the homomorphism which sends each of $x_{M+1}, \ldots, x_{N}$ to zero and other $x_{i}$ to themselves. It is clear that $\varphi_{N, M}\left(\Lambda_{N}\right)=\Lambda_{M}$ so we can consider the inverse limits in the category of graded algebras

$$
P=\lim _{\longleftarrow} P_{N}, \quad \Lambda=\lim _{\longleftarrow} \Lambda_{N}
$$

This means that

$$
\begin{array}{ll}
P=\oplus_{r=0}^{\infty} P^{r}, & P^{r}=\lim _{\longleftarrow} P_{N}^{r} \\
\Lambda=\oplus_{r=0}^{\infty} \Lambda^{r}, & \Lambda^{r}=\longleftarrow \lim _{\longleftarrow} \Lambda_{N}^{r}
\end{array}
$$

where $P_{N}^{r}, \Lambda_{N}^{r}$ are the homogeneous components of $P_{N}, \Lambda_{N}$ of degree $r$. The elements of $\Lambda$ are called symmetric functions. Since for any partition $\lambda$

$$
\varphi_{N, M}\left(m_{\lambda}\left(x_{1}, \ldots, x_{N}\right)\right)=m_{\lambda}\left(x_{1}, \ldots, x_{M}\right)
$$

(and similarly for the power sums) we can define the symmetric functions $m_{\lambda}, p_{\lambda}$.

Another important example of symmetric functions are Macdonald polynomials $P_{\lambda}(x, q, t)$. We give here their definition in the form most suitable for us.

Recall that on the set of partitions $\mathcal{P}_{N}$ there is the following dominance partial ordering: we write $\mu \leq \lambda$ if for all $i \geq 1$

$$
\mu_{1}+\mu_{2}+\cdots+\mu_{i} \leq \lambda_{1}+\lambda_{2}+\cdots+\lambda_{i} .
$$

Consider the following Macdonald-Ruijsenaars operator (MR operator)

$$
\mathcal{M}_{q, t}^{(N)}=\frac{1}{1-q} \sum_{i=1}^{N} \prod_{j \neq i} \frac{x_{i}-t x_{j}}{x_{i}-x_{j}}\left(T_{q, x_{i}}-1\right)
$$

where $T_{q, x_{i}}$ is the shift operator

$$
\left(T_{q, x_{i}} f\right)\left(x_{1}, \ldots, x_{i}, \ldots, x_{N}\right)=f\left(x_{1}, \ldots, q x_{i}, \ldots, x_{N}\right)
$$

This operator is related to the operators $D_{N}^{1}$ and $E_{N}$ from Macdonald's book [4] by the simple formulas

$$
\mathcal{M}_{q, t}^{(N)}=\frac{t^{N-1}}{1-q} D_{N}^{1}\left(q, t^{-1}\right)-\frac{1-t^{N}}{(1-q)(1-t)}=\frac{t^{-1}}{1-q} E_{N}\left(q, t^{-1}\right) .
$$

Our choice of the additional coefficient $\frac{1}{1-q}$ in formula (3) was motivated by the symmetric form of the deformed operator (1). We should note also that the operator (3) is related in a simple way to the trigonometric version of the operator $\hat{S}_{1}$ introduced by Ruijsenaars [3]. 
An important property of the MR operator is its stability under the change of $N$ : the following diagram is commutative

$$
\begin{array}{ll}
\Lambda_{N} \stackrel{\mathcal{M}_{q, t}^{(N)}}{\longrightarrow} & \Lambda_{N} \\
\downarrow \varphi_{N, M} & \downarrow \varphi_{N, M} \\
\Lambda_{M} \stackrel{\mathcal{M}_{q, t}^{(M)}}{\longrightarrow} & \Lambda_{M}
\end{array}
$$

(see page 321 in [4]). This allows us to define the MR operator $\mathcal{M}_{q, t}$ on the space of symmetric functions $\Lambda$ as the inverse limit of $\mathcal{M}_{q, t}^{(N)}$.

Recall [4] that Macdonald polynomials $P_{\lambda}(x, q, t) \in \Lambda_{N}$ are uniquely defined for generic parameters $q, t$ and any partition $\lambda, l(\lambda) \leq N$ by the following properties:

1) $P_{\lambda}(x, q, t)=m_{\lambda}+\sum_{\mu<\lambda} u_{\lambda \mu} m_{\mu}$, where $u_{\lambda \mu}=u_{\lambda \mu}(q, t) \in \mathbb{C}$

2) $P_{\lambda}(x, q, t)$ is an eigenfunction of the MR operator $\mathcal{M}_{q, t}^{(N)}$.

Indeed the operator $\mathcal{M}_{q, t}^{(N)}$ has an upper triangular matrix in the monomial basis $m_{\mu}$ :

$$
\mathcal{M}_{q, t}^{(N)}\left(m_{\lambda}\right)=\sum_{\mu \leq \lambda} c_{\lambda \mu} m_{\mu}
$$

where the coefficients $c_{\lambda \mu}$ can be described explicitly (see [4], page 321). In particular

$$
c_{\lambda, \lambda}=\frac{1}{1-q} \sum_{i=1}^{N}\left(q^{\lambda_{i}}-1\right) t^{i-1} .
$$

For generic parameters $q, t$ the coefficients $c_{\lambda, \lambda} \neq c_{\mu, \mu}$ for all $\lambda \neq \mu$ with $|\lambda|=|\mu|$, so the operator $\mathcal{M}_{q, t}^{(N)}$ is diagonalisable.

We should note that the coefficient $u_{\lambda \mu}$ are rational functions of $q$ and $t$, which have the singularities only if $q^{a}=t^{b}$ for some non-negative integers $a, b$ (not equal to zero simultaneously) [4]. Such parameters are called special, the Macdonald polynomials are well-defined for all non-special values of the parameters $q, t$.

From the stability of the MR operators it follows that

$$
\varphi_{N, M}\left(P_{\lambda}\left(x_{1}, \ldots, x_{N}\right)\right)=P_{\lambda}\left(x_{1}, \ldots, x_{M}\right)
$$

so we have correctly defined Macdonald symmetric functions $P_{\lambda}(x, q, t) \in \Lambda$ which are the eigenfunctions of the MR operator $\mathcal{M}_{q, t}^{(N)}$.

\section{Shifted Symmetric FunCtions And Shifted Macdonald POLYNOMIALS.}

We discuss now the so-called shifted Macdonald polynomials investigated by Knop, Sahi and Okounkov [5, 6, 7, 8].

Let us denote by $\Lambda_{N, t}$ the algebra of polynomials $f\left(x_{1}, \ldots, x_{N}\right)$ which are symmetric in the "shifted" variables $x_{i} t^{i-1}$. This algebra has the filtration 
by the degree of polynomials:

$$
\left(\Lambda_{N, t}\right)_{0} \subset\left(\Lambda_{N, t}\right)_{1} \subset \cdots \subset\left(\Lambda_{N, t}\right)_{r} \subset \cdots
$$

We have the following shifted analog of power sums:

$$
p_{r}^{*}\left(x_{1}, \ldots, x_{N}, t\right)=\sum_{i=1}^{N}\left(x_{i}^{r}-1\right) t^{r(i-1)}
$$

The polynomials

$$
p_{\lambda}^{*}(x, t)=p_{\lambda_{1}}^{*}(x, t) p_{\lambda_{2}}^{*}(x, t) \ldots,
$$

where $\lambda=\left(\lambda_{1}, \lambda_{2}, \ldots, \lambda_{r} \ldots\right)$ are partitions of length $l(\lambda) \leq N$, form a basis in $\Lambda_{N, t}$. They are stable in the following sense. Let $M \leq N$ and $\varphi_{N, M}^{*}: P_{N} \longrightarrow P_{M}$ be the homomorphism which sends each of $x_{M+1}, \ldots, x_{N}$ to 1 and leaving the remaining $x_{i}$ unchanged. Then $\varphi_{N, M}^{*}\left(p_{\lambda}^{*}\left(x_{1}, \ldots, x_{N}\right)\right)=$ $p_{\lambda}^{*}\left(x_{1}, \ldots, x_{M}\right)$. Therefore $\varphi_{N, M}^{*}\left(\Lambda_{N, t}\right)=\Lambda_{M, t}$ and one can consider the inverse limit

$$
\Lambda_{t}=\lim _{\longleftarrow} \Lambda_{N, t}
$$

in the category of filtered algebras:

$$
\left.\Lambda_{t}=\bigcup_{r=0}^{\infty}\left(\Lambda_{t}\right)_{r}, \quad\left(\Lambda_{t}\right)_{r}=\longleftarrow \Lambda_{N, t}\right)_{r} .
$$

The algebra $\Lambda_{t}$ is called the algebra of shifted symmetric functions $[7,8]$. Let us introduce the following function on the set of partitions:

$$
H(\lambda, q, t)=t^{n\left(\lambda^{\prime}\right)} q^{n(\lambda)} \prod_{s \in \lambda}\left(q^{a(s)+1}-t^{l(s)}\right) .
$$

Here $a(s)$ and $l(s)$ are the arm and leg lengths respectively of a box $s=$ $(i, j) \in \lambda$, which are defined

$$
a(s)=\lambda_{i}-j, \quad l(s)=\lambda_{j}^{\prime}-i
$$

and

$$
n(\lambda)=\sum_{i \geq 1}(i-1) \lambda_{i}
$$

Recall (see $[6,7,8])$ that the shifted Macdonald polynomial $P_{\lambda}^{*}(x, q, t) \in \Lambda_{t}$ is the unique shifted symmetric function of degree $\operatorname{deg} P_{\lambda}=|\lambda|$ satisfying the following property:

$$
P_{\lambda}^{*}\left(q^{\lambda}, q, t\right)=H(\lambda, q, t)
$$

and $P_{\lambda}^{*}\left(q^{\mu}, q, t\right)=0$ unless $\lambda \subseteq \mu$ (Extra Vanishing Condition). Here and later throughout the paper by $P\left(q^{\lambda}\right)$ for a partition $\lambda=\left(\lambda_{1}, \ldots, \lambda_{N}\right)$ we mean $P\left(q^{\lambda_{1}}, \ldots, q^{\lambda_{N}}, 1,1, \ldots\right)$.

We will need the following duality property of the shifted Macdonald polynomials proved by Okounkov [8]

$$
P_{\lambda}^{*}\left(q^{\mu}, q, t\right)=\frac{H(\lambda, q, t)}{H\left(\lambda^{\prime}, t, q\right)} P_{\lambda^{\prime}}^{*}\left(t^{\mu^{\prime}}, t, q\right) .
$$


To show this consider the following conjugation homomorphism (cf. [2]):

$$
\left[\left(\omega_{q, t}^{*}(f)\right)\right]\left(t^{\lambda}\right)=f\left(q^{\lambda^{\prime}}\right)
$$

We claim that the conjugation homomorphism maps the algebra of shifted symmetric functions $\Lambda_{t}$ into the algebra $\Lambda_{q}$. Indeed computing the sum

$$
\sum_{(i, j) \in \lambda} q^{i-1} t^{j-1}
$$

first along columns and then along the rows we come to the following equality

$$
\frac{1}{1-q} \sum_{j \geq 1}\left(q^{\lambda_{j}^{\prime}}-1\right) t^{j-1}=\frac{1}{1-t} \sum_{i \geq 1}\left(t^{\lambda_{i}}-1\right) q^{i-1}
$$

which is equivalent to

$$
\left(\omega_{q, t}^{*}\left(p_{r}^{*}(x, t)\right)=\frac{1-q^{r}}{1-t^{r}} p_{r}^{*}(x, q)\right.
$$

with $r=1$. Replacing $q$ by $q^{r}$ and $t$ by $t^{r}$ we have this formula for all $r$. Now the claim follows from the fact that $p_{\lambda}^{*}(x, t)$ generate the algebra.

Combining this with the definition of the shifted Macdonald polynomials we have the duality property (6).

\section{Cherednik - Dunkl operators and Harish-Chandra HOMOMORPHISM}

In this section we present the basic facts about Cherednik-Dunkl operators. For more details we refer to $[12,13]$.

Consider the operators $T_{i}, i=1, \ldots, N-1$

$$
T_{i}=1+\frac{x_{i}-t x_{i+1}}{x_{i}-x_{i+1}}\left(\sigma_{i i+1}-1\right)
$$

and

$$
\omega=\sigma_{N N-1} \sigma_{N-1 N-2} \ldots \sigma_{21} T_{q, x_{1}}
$$

where $\sigma_{i j}$ is acting on the function $f\left(x_{1}, \ldots, x_{N}\right)$ by permutation of the $i$ th and $j$-th coordinates. By Cherednik-Dunkl operators we will mean the following difference operators

$$
D_{i, N}=t^{1-N} T_{i} \ldots T_{N-1} \omega T_{1}^{-1} \ldots T_{i-1}^{-1}, \quad i=1, \ldots N .
$$

The first important property of the Cherednik-Dunkl operators is that they commute with each other:

$$
\left[D_{i, N}, D_{j, N}\right]=0 .
$$

This means that one can substitute them in any polynomial $P$ in $N$ variables without ordering problems.

The second property is that if one does this for a shifted symmetric polynomial $g \in \Lambda_{N, t}$ then the corresponding operator $g\left(D_{1, N} \ldots D_{N, N}\right)$ leaves the algebra of symmetric polynomials $\Lambda_{N}$ invariant:

$$
g\left(D_{1, N} \ldots D_{N, N}\right): \Lambda_{N} \rightarrow \Lambda_{N}
$$


The restriction of the operator $g\left(D_{1, N} \ldots D_{N, N}\right)$ on the algebra $\Lambda_{N}$ is given by some difference operator, which we will denote as $\mathcal{D}_{N, q, t}^{g}$.

One can check that if we apply this operation to the shifted power sum $p_{1}^{*}\left(x_{1}, \ldots, x_{N}, t\right)=\sum_{i=1}^{N}\left(x_{i}-1\right) t^{i-1}$ we arrive (up to a factor $(1-q)^{-1}$ ) at Macdonald-Ruijsenaars operator (3). Thus the operators $\mathcal{D}_{N, q, t}^{g}$ can be considered as the integrals of the corresponding quantum system, which is equivalent to the relativistic Calogero-Moser system introduced by Ruijsenaars [3].

The Macdonald polynomials are the joint eigenfunctions of all these operators: if $P_{\lambda}(x, q, t)$ is the Macdonald polynomial corresponding a partition $\lambda$ of weight $N$ then

$$
\mathcal{D}_{N, t}^{g} P_{\lambda}(x, q, t)=g\left(q^{\lambda_{1}}, q^{\lambda_{2}}, \ldots, q^{\lambda_{N}}\right) P_{\lambda}(x, q, t)
$$

This allows us to define a homomorphism (which is actually a monomorphism) $\chi: f \rightarrow \mathcal{D}_{N, t}^{f}$ from the algebra $\Lambda_{t}$ to the algebra of difference operators. Let us denote by $\mathcal{D}(N, t)$ the image of $\chi$. The inverse homomorphism

$$
\chi^{-1}: \mathcal{D}(N, t) \longrightarrow \Lambda_{N, t}
$$

is called the Harish-Chandra isomorphism. It can be defined by its action on the Macdonald polynomials: the image of $\mathcal{D} \in \mathcal{D}(N, t)$ is a polynomial $f=f_{\mathcal{D}} \in \Lambda_{N, t}$ such that

$$
\mathcal{D} P_{\lambda}(x, q, t)=f\left(q^{\lambda}\right) P_{\lambda}(x, q, t) .
$$

One can check that the Cherednik-Dunkl operators are stable: the diagram

$$
\begin{array}{ll}
P_{N} \stackrel{D_{i, N}}{\longrightarrow} & P_{N} \\
\downarrow \varphi_{N, M} & \downarrow \varphi_{N, M} \\
P_{M} \stackrel{D_{i, M}}{\longrightarrow} & P_{M}
\end{array}
$$

is commutative for all $M \leq N$ and $i \geq 1$. Similarly for any $f \in \Lambda_{N, t}$ and $g=\varphi_{N, M}^{*}(f), M \leq N$ the following diagram is commutative:

$$
\begin{array}{ll}
\Lambda_{N} \stackrel{\mathcal{D}_{N, t}^{f}}{\longrightarrow} & \Lambda_{N} \\
\downarrow \varphi_{N, M} & \downarrow \varphi_{N, M} \\
\Lambda_{M} \stackrel{\mathcal{D}_{M, t}^{g}}{\longrightarrow} & \Lambda_{M}
\end{array}
$$

This allows us to define for any shifted symmetric function $f \in \Lambda_{t}$ a difference operator

$$
\mathcal{D}_{t}^{f}: \Lambda \longrightarrow \Lambda
$$

and the infinite dimensional version of the homomorphism $\chi$. We will denote by $\mathcal{D}(t)$ the image of this homomorphism. The inverse (Harish-Chandra) homomorphism $\chi^{-1}: \mathcal{D}(t) \longrightarrow \Lambda_{t}$ can be described by the relation

$$
\mathcal{D}_{t}^{f} P_{\lambda}(x, q, t)=f\left(q^{\lambda}\right) P_{\lambda}(x, q, t),
$$

where now $f \in \Lambda_{t}$ and $P_{\lambda}(x, q, t)$ are Macdonald polynomials. 


\section{Deformed Macdonald-RuiJsenaArs operator as a Restriction}

The following algebra $\Lambda_{n, m, q, t}$ will play a central role in our construction. Let $P_{n, m}=\mathbb{C}\left[x_{1}, \ldots, x_{n}, y_{1}, \ldots, y_{m}\right]$ be the polynomial algebra in $n+m$ independent variables. Then $\Lambda_{n, m, q, t} \subset P_{n, m}$ is the subalgebra consisting of polynomials which are symmetric in $x_{1}, \ldots, x_{n}$ and $y_{1}, \ldots, y_{m}$ separately and satisfy the conditions

$$
T_{q, x_{i}}(f)=T_{t, y_{j}}(f)
$$

on each hyperplane $x_{i}-y_{j}=0$ for all $i=1, \ldots, n$ and $j=1, \ldots, m$.

Assume from now on that $t$ and $q$ are not roots of unity and consider the following deformed Newton sums

$$
p_{r}(x, y, q, t)=\sum_{i=1}^{n} x_{i}^{r}+\frac{1-q^{r}}{1-t^{r}} \sum_{j=1}^{m} y_{j}^{r},
$$

which obviously belong to $\Lambda_{n, m, q, t}$ for all nonnegative integers $r$.

We will prove later that if the parameters $q, t$ are non-special, then $\Lambda_{n, m ; q, t}$ is generated by the deformed Newton sums $p_{r}(x, y, q, t)$ (see Theorem 5.8 below), but now let us start with the following result.

Theorem 5.1. The algebra $\Lambda_{n, m, q, t}$ is finitely generated if and only if $t^{i} q^{j} \neq$ 1 for all $1 \leq i \leq n, 1 \leq j \leq m$.

Proof. Consider the subalgebra $P(k)=\mathbf{C}\left[p_{1}, \ldots, p_{n+m}\right]$ generated by the first $n+m$ deformed Newton sums (12). We need the following result about common zeros of these polynomials (cf. Proposition 4 in [1]).

Lemma 5.2. The system

$$
\left\{\begin{aligned}
x_{1}+x_{2}+\cdots+x_{n}+\frac{1-q}{1-t}\left(x_{n+1}+x_{n+2}+\cdots+x_{n+m}\right)= & 0 \\
x_{1}^{2}+x_{2}^{2}+\cdots+x_{n}^{2}+\frac{1-q^{2}}{1-t^{2}}\left(x_{n+1}^{2}+x_{n+2}^{2}+\cdots+x_{n+m}^{2}\right)= & 0 \\
& \vdots \\
x_{1}^{n+m}+x_{2}^{n+m}+\cdots+x_{n}^{n+m}+\frac{1-q^{n+m}}{1-t^{n+m}}\left(x_{n+1}^{n+m}+x_{n+2}^{n+m}+\cdots+x_{n+m}^{n+m}\right)= & 0
\end{aligned}\right.
$$

has a non-zero solution in $\mathbf{C}^{n+m}$ if and only if $t^{i} q^{j}=1$ for some $1 \leq i \leq$ $n, 1 \leq j \leq m$.

Proof. Let us multiply the $k$-th equation by $1-t^{k}$ and rewrite it as $x_{1}^{k}+\cdots+x_{n}^{k}+x_{n+1}^{k}+\cdots+x_{n+m}^{k}=\left(t x_{1}\right)^{k}+\cdots+\left(t x_{n}\right)^{k}+\left(q x_{n+1}\right)^{k}+\cdots+\left(q x_{n+m}\right)^{k}$.

Since this is true for all $k=1, \ldots, n+m$ this means that the set

$$
x_{1}, \ldots, x_{n}, x_{n+1}, \ldots, x_{n+m}
$$

coincides up to a permutation with the set

$$
t x_{1}, \ldots, t x_{n}, q x_{n+1}, \ldots, q x_{n+m} .
$$


Let us consider only the nonzero elements $x_{i}, i \in S \subset[1, \ldots, n]$ and the nonzero elements $x_{n+j}, j \in T \subset[1, \ldots, m]$. Therefore

$$
\prod_{i \in S} x_{i} \prod_{j \in T} x_{n+j}=t^{|S|} q^{|T|} \prod_{i \in S} x_{i} \prod_{j \in T} x_{n+j}
$$

Therefore $t^{|S|} q^{|T|}=1$.

Conversely suppose that $t^{i} q^{j}=1$ for some $1 \leq i \leq n, 1 \leq j \leq m$ and consider

$$
x_{1}=1, x_{2}=t^{-1}, \ldots, x_{i}=t^{1-i}, \quad x_{n+1}=q^{-1} t^{1-i}, \ldots, x_{n+j}=q^{-j} t^{1-i}
$$

with every other variable set to zero. Then it is easy to verify that it is a solution of the system.

From the lemma and Nullstellensatz it follows that if $t^{i} q^{j} \neq 1$ for all $1 \leq i \leq n, 1 \leq j \leq m$ the elements $x_{i}^{N_{i}}$ belong to the ideal generated by $p_{1}, \ldots, p_{n+m}$ for some $N_{i}$ and all $i=1, \ldots, n+m$. By a standard result from commutative algebra (see Corollary 5.2 in [15]) it follows that the algebra $P_{n, m}$ is a finitely generated module over subalgebra $P(k)$. Now using Proposition 7.8 from [15] we conclude that the algebra $\Lambda_{n, m, q, t}$ in this case is finitely generated.

Conversely, assume that $t^{i} q^{j}=1$ for some $1 \leq i \leq n, 1 \leq j \leq m$ and consider the following homomorphism

$$
\Phi_{i, j}: \Lambda_{n, m ; q, t} \rightarrow \mathbb{C}[u, v]
$$

sending a polynomial $f\left(x_{1}, \ldots, x_{n}, y_{1}, \ldots, y_{m}\right)$ into

$$
\phi(u, v)=f\left(u, t^{-1} u, \ldots, t^{1-i} u, 0, \ldots, 0, q^{j-1} t v, q^{j-2} t v, \ldots, t v, 0, \ldots, 0\right) .
$$

One can check that the image $\phi$ of any $f \in \Lambda_{n, m ; q, t}$ satisfies the condition $\phi(u, u)=\phi(q u, q u)$ and therefore $\phi(u, u)=$ const since by assumption $q$ is not a root of unity. Moreover one can show that any such function $\phi$ belongs to the image of $\Phi_{i, j}$. The corresponding algebra consists of the polynomials of the form $\phi=(u-v) p(u, v)+c$, which is not finitely generated (c.f. [1], p. 274). This completes the proof of the theorem.

Let us assume from now on that $q, t$ are generic. Since the algebra $\Lambda_{n, m, q, t}$ is finitely generated we can introduce an affine algebraic variety

$$
\Delta_{n, m, q, t}=\operatorname{Spec} \Lambda_{n, m, q, t} .
$$

Consider the following embedding of $\Delta_{n, m, q, t}$ into infinite-dimensional Macdonald variety $\mathcal{M}=\operatorname{Spec} \Lambda$ (cf. [2]). Recall that $\Lambda$ is the algebra of symmetric functions in an infinite number of variables $z_{1}, z_{2}, \ldots$, which is freely generated by the powers sums $p_{r}(z)=z_{1}^{r}+z_{2}^{r}+\ldots$ Consider the following homomorphism $\varphi$ from $\Lambda$ to $\Lambda_{n, m, q, t}$ uniquely determined by the relations

$$
\varphi\left(p_{r}(z)\right)=p_{r}(x, y, q, t) .
$$

For generic $q, t$ this homomorphism is surjective and thus defines an embed$\operatorname{ding} \phi: \Delta_{n, m, q, t} \rightarrow \mathcal{M}$. 
We are going to show that the deformed MR operator (1) is the restriction of the usual MR operator on $\mathcal{M}$ onto the subvariety $\Delta_{n, m, q, t}$.

We start with the following modification of Proposition 2.8 from paper [10] by Chalykh.

Proposition 5.3. The deformed $M R$ operator preserves the algebra $\Lambda_{n, m, q, t}$ :

$$
\mathcal{M}_{n, m, q, t}: \Lambda_{n, m, q, t} \rightarrow \Lambda_{n, m, q, t} .
$$

Proof. Let $f \in \Lambda_{n, m, q, t}$ and $g=\mathcal{M}_{n, m, q, t}(f)$. Then we have

$$
g=\sum_{i=1}^{n} \frac{A_{i}}{1-q} f_{i}+\sum_{j=1}^{m} \frac{B_{j}}{1-t} f_{\bar{j}}
$$

where $A_{i}, B_{j}$ are the same as in (1) and

$$
f_{i}=T_{q, x_{i}}(f)-f, \quad f_{\bar{j}}=T_{t, y_{j}}(f)-f, \quad i=1, \ldots, n, j=1, \ldots, m .
$$

Let us prove first that $g$ is a polynomial. It is clear that $g$ is a rational function, which is symmetric in $x_{1}, \ldots, x_{n}$ and $y_{1}, \ldots, y_{m}$, so, given the form of $A_{i}$ and $B_{j}$, it is enough to prove that $g$ has no singularities of the type $\left(x_{1}-x_{2}\right)^{-1},\left(y_{1}-y_{2}\right)^{-1},\left(x_{1}-y_{1}\right)^{-1}$.

Let us represent $g$ in the form

$$
g=\frac{1}{x_{1}-x_{2}}\left(\left(x_{1}-x_{2}\right) \frac{A_{1}}{1-q} f_{1}+\left(x_{1}-x_{2}\right) \frac{A_{2}}{1-q} f_{2}\right)+g_{1},
$$

where $g_{1}$ is a rational function without singularities on the hyperplane $x_{1}=$ $x_{2}$. But on this hyperplane we have

$\left(x_{1}-x_{2}\right) \frac{A_{1}}{1-q} f_{1}+\left(x_{1}-x_{2}\right) \frac{A_{2}}{1-q} f_{2}=\left(\left(x_{1}-x_{2}\right) \frac{A_{1}}{1-q}+\left(x_{1}-x_{2}\right) \frac{A_{2}}{1-q}\right) f_{1}=0$,

so we see that $g$ has no poles when $x_{1}=x_{2}$. Similarly there are no singularities of type $\left(y_{1}-y_{2}\right)^{-1}$. Now write $g$ in the form

$$
g=\frac{1}{x_{1}-y_{1}}\left(\left(x_{1}-y_{1}\right) \frac{A_{1}}{1-q} h_{1}+\left(x_{1}-y_{1}\right) \frac{B_{1}}{1-t} f_{\overline{1}}\right)+g_{2},
$$

where $g_{2}$ is a rational function without poles when $x_{1}=y_{1}$. On the hyperplane $x_{1}=y_{1}$ we have

$$
\left(x_{1}-y_{1}\right) \frac{A_{1}}{1-q}+\left(x_{1}-y_{1}\right) \frac{B_{1}}{1-t}=0,
$$

therefore

$\left(x_{1}-y_{1}\right) \frac{A_{1}}{1-q} f_{1}+\left(x_{1}-y_{1}\right) \frac{B_{1}}{1-t} f_{\overline{1}}=\left(\left(x_{1}-y_{1}\right) \frac{A_{1}}{1-q}+\left(x_{1}-y_{1}\right) \frac{B_{1}}{1-t}\right) f_{1}=0$.

We have used here the fact that when $x_{1}=y_{1}$ we have $f_{1}=f_{\overline{1}}$ from the definition of algebra $\Lambda_{n, m, q, t}$. Thus we have proved that $g$ is a polynomial.

Now let us prove that $g \in \Lambda_{n, m, q, t}$. On the hyperplane $x_{1}=y_{1}$ we have the following equalities:

$$
\left(T_{q, x_{1}}-T_{t, y_{1}}\right) A_{i}=0, \quad i \neq 1, \quad T_{q, x_{1}} A_{1}=0,
$$




$$
\begin{gathered}
\left(T_{q, x_{1}}-T_{t, y_{1}}\right) B_{j}=0, \quad j \neq 1 \quad T_{t, y_{1}} B_{1}=0 \\
T_{t, y_{1}} \frac{A_{1}}{1-q}=T_{q, x_{1}} \frac{B_{1}}{1-t},
\end{gathered}
$$

and therefore

$$
\begin{gathered}
\left(T_{q, x_{1}}-T_{t, y_{1}}\right) g=\left(T_{q, x_{1}}-T_{t, y_{1}}\right)\left(\frac{A_{1}}{1-q} f_{1}+\frac{B_{1}}{1-t} f_{\overline{1}}\right)= \\
T_{q, x_{1}}\left(\frac{B_{1}}{1-t} f_{\overline{1}}\right)-T_{t, y_{1}}\left(\frac{A_{1}}{1-q} f_{1}\right)=T_{q, x_{1}}\left(\frac{B_{1}}{1-t}\right)\left(T_{q, x_{1}} T_{t, y_{1}}(f)-T_{q, x_{1}} f\right)- \\
T_{t, y_{1}}\left(\frac{A_{1}}{1-q}\right)\left(T_{t, y_{1}} T_{q, x_{1}}(f)-T_{t, y_{1}} f\right)=0
\end{gathered}
$$

since $f \in \Lambda_{n, m, q, t}$.

Now we are ready to formulate our central result. Let $\mathcal{M}_{q, t}$ be the usual MR operator in infinite dimension.

Theorem 5.4. The following diagram is commutative for all values of the parameters $q, t$ :

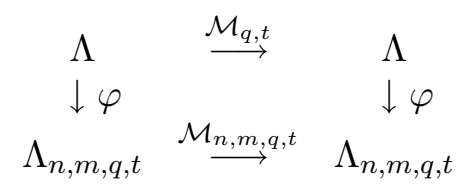

In other words, the deformed $M R$ operator (1) is the restriction of the operator $\mathcal{M}_{q, t}$ onto the subvariety $\Delta_{n, m, q, t} \subset \mathcal{M}$.

Proof. Let us introduce the following function $\Pi \in \Lambda\left[\left[w_{1}, \ldots, w_{N}\right]\right]$ which plays an important role in the theory of Macdonald polynomials (see [4]):

$$
\Pi=\prod_{j=1}^{N} \prod_{r=0}^{\infty} \prod_{i=1}^{\infty} \frac{1-t^{-1} q^{r} z_{i} w_{j}}{1-q^{r} z_{i} w_{j}}
$$

Lemma 5.5. The function $\Pi$ satisfies the following properties:

(i)

$$
\mathcal{M}_{q, t}^{z} \Pi=\mathcal{M}_{q, t}^{w} \Pi,
$$

where index $z$ (resp. $w$ ) indicates the action of the Macdonald operator $\mathcal{M}_{q, t}$ on $z$ (resp. $w$ ) variables

(ii)

$$
\varphi(\Pi)=\prod_{r=0}^{\infty} \prod_{i=1}^{n} \prod_{l=1}^{N} \frac{1-t^{-1} q^{r} x_{i} w_{l}}{1-q^{r} x_{i} w_{l}} \prod_{j=1}^{m} \prod_{l}\left(1-t^{-1} y_{j} w_{l}\right)
$$

(iii)

$$
\varphi\left(\mathcal{M}_{q, t}^{z} \Pi\right)=\mathcal{M}_{n, m, q, t} \varphi(\Pi)
$$


Proof. The first part is well known, see Macdonald [4], formula (3.12) in Chapter 6.

To prove the second one we note that since $\varphi$ is a homomorphism it is enough to consider the case $N=1$ when we have only one variable $w$. Consider an auxiliary homomorphism

$$
\tilde{\varphi}: \Lambda \rightarrow \Lambda_{n} \otimes \Lambda
$$

defined by

$$
\tilde{\varphi}\left(p_{r}(z)\right)=p_{r}(x)+\frac{1-q^{r}}{1-t^{r}} p_{r}(y)
$$

with finite number $n$ of variables $x_{i}$ and infinite number of variables $y_{i}$. Define also the following automorphism $\sigma_{q, t}: \Lambda \rightarrow \Lambda$ by

$$
\sigma_{q, t}\left(p_{r}(y)\right)=\frac{1-q^{r}}{1-t^{r}} p_{r}(y)
$$

We have (cf. [2])

$$
\begin{gathered}
\tilde{\varphi}(\Pi)=\prod_{r=0}^{\infty} \prod_{i=1}^{n} \frac{1-t^{-1} q^{r} x_{i} w}{1-q^{r} x_{i} w} \sigma_{q, t}\left(\prod_{r=0}^{\infty} \prod_{j \geq 1} \frac{1-t^{-1} q^{r} y_{j} w}{1-q^{r} y_{j} w}\right) \\
=\prod_{r=0}^{\infty} \prod_{i=1}^{n} \frac{1-t^{-1} q^{r} x_{i} w}{1-q^{r} x_{i} w} \sigma_{q, t}\left(\exp \log \prod_{r=0}^{\infty} \prod_{j \geq 1} \frac{1-t^{-1} q^{r} y_{j} w}{1-q^{r} y_{j} w}\right) \\
=\prod_{r=0}^{\infty} \prod_{i=1}^{n} \frac{1-t^{-1} q^{r} x_{i} w}{1-q^{r} x_{i} w} \exp \sigma_{q, t}\left(\log \prod_{r=0}^{\infty} \prod_{j \geq 1} \frac{1-t^{-1} q^{r} y_{j} w}{1-q^{r} y_{j} w}\right) \\
=\prod_{r=0}^{\infty} \prod_{i=1}^{n} \frac{1-t^{-1} q^{r} x_{i} w}{1-q^{r} x_{i} w} \exp \sigma_{q, t}\left(\sum_{r=0}^{\infty} \sum_{j \geq 1} \log \left(1-t^{-1} q^{r} y_{j} w\right)-\log \left(1-q^{r} y_{j} w\right)\right. \\
=\prod_{r=0}^{\infty} \prod_{i=1}^{n} \frac{1-t^{-1} q^{r} x_{i} w}{1-q^{r} x_{i} w} \exp \sigma_{q, t}\left(\sum_{r=0}^{\infty} \sum_{j \geq 1} \sum_{s \geq 1} \frac{q^{r s} y_{j}^{s} w^{s}-t^{-s} q^{r s} y_{j}^{s} w^{s}}{s}\right) \\
=\prod_{r=0}^{\infty} \prod_{i=1}^{n} \frac{1-t^{-1} q^{r} x_{i} w}{1-q^{r} x_{i} w} \exp \sigma_{q, t}\left(\sum_{s \geq 1} \frac{1-t^{-s}}{1-q^{s}} p_{s}(y) \frac{w^{s}}{s}\right) \\
=\prod_{r=0}^{\infty} \prod_{i=1}^{n} \frac{1-t^{-1} q^{r} x_{i} w}{1-q^{r} x_{i} w} \exp \left(\sum_{s \geq 1} \frac{1-t^{-s}}{1-t^{s}} p_{s}(y) \frac{w^{s}}{s}\right) \\
=\prod_{r=0}^{\infty} \prod_{i=1}^{n} \frac{1-t^{-1} q^{r} x_{i} w}{1-q^{r} x_{i} w} \exp \left(-\sum_{s \geq 1} t^{-s} p_{s}(y) \frac{w^{s}}{s}\right) \\
=\prod_{r=0}^{\infty} \prod_{i=1}^{n} \frac{1-t^{-1} q^{r} x_{i} w}{1-q^{r} x_{i} w} \prod_{j=1}^{\infty}\left(1-t^{-1} y_{j} w\right) .
\end{gathered}
$$


Now setting all the variables $y_{i}$ except the first $m$ to zero we have the second part of the Lemma.

Let us prove $(\mathrm{iii})$. It is easy to check the following equalities

$$
\begin{gathered}
\varphi(\Pi)^{-1} T_{q, w_{l}} \varphi(\Pi)=\prod_{i=1}^{n} \frac{1-x_{i} w_{l}}{1-t^{-1} x_{i} w_{l}} \prod_{j=1}^{m} \frac{1-t^{-1} q y_{j} w_{l}}{1-t^{-1} y_{j} w_{l}}, \\
\varphi(\Pi)^{-1} T_{q, x_{i}} \varphi(\Pi)=\prod_{l=1}^{N} \frac{1-x_{i} w_{l}}{1-t^{-1} x_{i} w_{l}}, \\
\varphi(\Pi)^{-1} T_{q, y_{j}} \varphi(\Pi)=\prod_{l=1}^{N} \frac{1-y_{j} w_{l}}{1-t^{-1} y_{j} w_{l}} .
\end{gathered}
$$

Now we see that $(i i i)$ is equivalent to the following equality

$$
\begin{gathered}
\sum_{l=1}^{N} C_{l}\left(\prod_{i=1}^{n} \frac{1-x_{i} w_{l}}{1-t^{-1} x_{i} w_{l}} \prod_{j=1}^{m} \frac{1-t^{-1} q y_{j} w_{l}}{1-t^{-1} y_{j} w_{l}}-1\right) \\
=\sum_{i=1}^{n} A_{i}\left(\prod_{l=1}^{N} \frac{1-x_{i} w_{l}}{1-t^{-1} x_{i} w_{l}}-1\right)+\frac{1-q}{1-t} \sum_{j=1}^{m} B_{j}\left(\prod_{l=1}^{N} \frac{1-t^{-1} q y_{j} w_{l}}{1-t^{-1} y_{j} w_{l}}-1\right),
\end{gathered}
$$

where

$$
C_{l}=\prod_{k \neq l}^{N} \frac{w_{l}-t w_{k}}{w_{l}-w_{k}}, A_{i}=\prod_{s \neq i}^{n} \frac{x_{i}-t x_{s}}{x_{i}-x_{s}} \prod_{j=1}^{m} \frac{x_{i}-q y_{j}}{x_{i}-y_{j}}, B_{j}=\prod_{r \neq j}^{m} \frac{y_{j}-q y_{r}}{y_{j}-y_{r}} \prod_{i=1}^{n} \frac{y_{j}-t x_{i}}{y_{j}-x_{i}} .
$$

From the partial fraction decomposition we have

$$
\begin{gathered}
\prod_{i=1}^{n} \frac{1-x_{i} w_{l}}{1-t^{-1} x_{i} w_{l}} \prod_{j=1}^{m} \frac{1-t^{-1} q y_{j} w_{l}}{1-t^{-1} y_{j} w_{l}}-1 \\
=t^{n} q^{m}-1+(1-t) \sum_{i=1}^{n} \frac{A_{i}}{1-t^{-1} x_{i} w_{l}}+(1-q) \sum_{j=1}^{m} \frac{B_{j}}{1-t^{-1} y_{j} w_{l}}, \\
\prod_{l=1}^{N} \frac{1-x_{i} w_{l}}{1-t^{-1} x_{i} w_{l}}-1=t^{N}-1+(1-t) \sum_{l=1}^{N} \frac{C_{l}}{1-t^{-1} x_{i} w_{l}}, \\
\prod_{l=1}^{N} \frac{1-y_{j} w_{l}}{1-t^{-1} y_{j} w_{l}}-1=t^{N}-1+(1-t) \sum_{l=1}^{N} \frac{C_{l}}{1-t^{-1} y_{j} w_{l}} .
\end{gathered}
$$

Substituting these identities into relation (19) we reduce it to the following equality

$$
\left(t^{n} q^{m}-1\right) \sum_{l=1}^{N} C_{l}=\left(t^{N}-1\right) \sum_{i=1}^{n} A_{i}+\frac{1-q}{1-t}\left(t^{N}-1\right) \sum_{j=1}^{m} B_{j}
$$




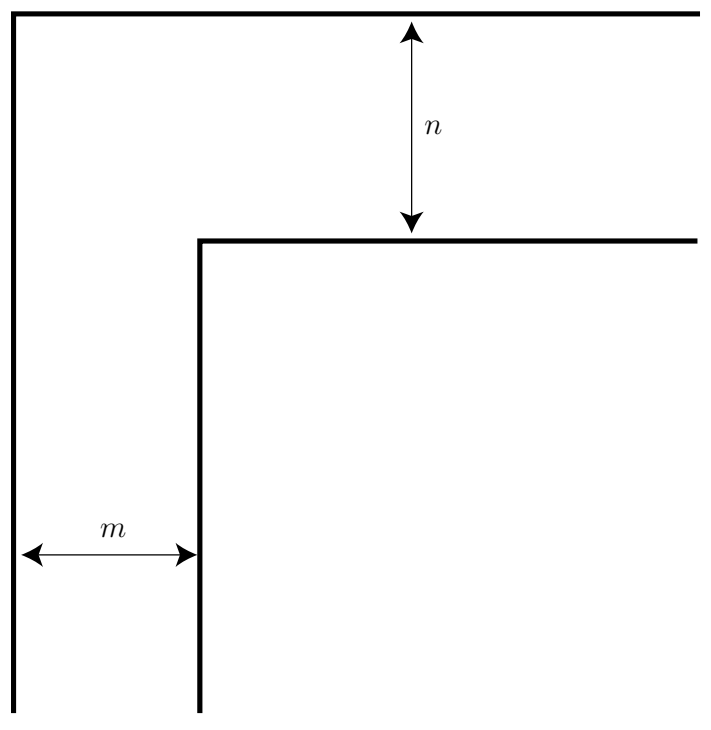

Fig. 1: Fat hook

which follows from the identities

$$
\sum_{l=1}^{N} C_{l}=\frac{t^{N}-1}{t-1}, \quad \sum_{i=1}^{n} A_{i}+\frac{1-q}{1-t} \sum_{j=1}^{m} B_{j}=\frac{t^{n} q^{m}-1}{t-1}
$$

The lemma is proved.

To complete the proof of the Theorem we need Macdonald's result that the coefficients $g_{\lambda}(z, q, t)$ in the expansion of the function

$$
\Pi=\prod_{j=1}^{N} \prod_{r=0}^{\infty} \prod_{i=1}^{\infty} \frac{1-t^{-1} q^{r} z_{i} w_{j}}{1-q^{r} z_{i} w_{j}}=\sum_{\lambda} g_{\lambda}(z, q, t) m_{\lambda}(w)
$$

linearly generate $\Lambda$ when we increase the number of variables $w$ (see [4], VI, $(2.10))$.

Let us introduce the set of partitions $H_{n, m}$, which consists of the partitions $\lambda$ such that $\lambda_{n+1} \leq m$ or, in other words, whose diagrams are contained in the fat $(n, m)$ - hook (see Fig.1). Its complement we will denote as $\bar{H}_{n, m}$.

Theorem 5.6. If $q, t$ are non-special then Ker $\varphi$ is spanned by the Macdonald polynomials $P_{\lambda}(z, q, t)$ corresponding to the partitions which are not contained in the fat $(n, m)$-hook.

Proof. Consider the automorphism $\sigma_{q, t}$ of algebra $\Lambda$ defined above by (18):

$$
\sigma_{q, t}\left(p_{r}\right)=\frac{1-q^{r}}{1-t^{r}} p_{r}
$$


Then using formula (6.19) from [4] (see page 327) it is easy to verify that

$$
\sigma_{q, t}\left(P_{\lambda}(z, q, t)\right)=(-1)^{|\mu|} \frac{H(\lambda, q, t)}{H\left(\lambda^{\prime}, t, q\right)} P_{\lambda^{\prime}}(z, t, q) .
$$

Let now $x=\left(x_{1}, x_{2}, \ldots\right), y=\left(y_{1}, y_{2}, \ldots,\right)$ be two infinite sequences. Then we have (see [4], page 345 , formula $\left(7.9^{\prime}\right)$ )

$$
P_{\lambda}(x, y, q, t)=\sum_{\mu \subset \lambda} P_{\lambda / \mu}(x, q, t) P_{\mu}(y, q, t),
$$

where $P_{\lambda / \mu}(z, q, t)$ are the skew Macdonald functions defined in [4] (see Chapter $6, \S 7$ ) and $\mu \subset \lambda$ means that $\mu_{i} \leq \lambda_{i}$ (or equivalently the diagram of $\mu$ is a subset of the diagram of $\lambda$ ).

If we apply this automorphism $\sigma_{q, t}$ acting in $y$ variables on both sides of the formula (21) and put all the variables $x$ and $y$ except the first $n$ and $m$ respectively to zero similarly to the proof of the second part of lemma 5.4 we get

$$
\varphi\left(P_{\lambda}(z, q, t)\right)=\sum_{\mu \subset \lambda}(-1)^{|\mu|} P_{\lambda / \mu}(x, q, t) \frac{H(\mu, q, t)}{H\left(\mu^{\prime}, t, q\right)} P_{\mu^{\prime}}(y, t, q) .
$$

Now let us assume that $\lambda$ is not contained in the fat $(n, m)$-hook, then $\lambda_{m+1}^{\prime}>n$. We have two possibilities: $\mu_{m+1}^{\prime}>0$ or $\mu_{m+1}^{\prime}=0$. In the first case we have $P_{\mu^{\prime}}\left(y_{1}, \ldots, y_{m}, t, q\right)=0$. In the second case we have $\lambda_{m+1}^{\prime}-\mu_{m+1}^{\prime}>n$, so according to [4] (page 347, formula (7.15)) the skew function $P_{\lambda / \mu}\left(x_{1}, \ldots, x_{n}, q, t\right)=0$. Thus we have shown that the Macdonald polynomials $P_{\lambda}(z, q, t)$ with $\lambda \in \bar{H}_{n, m}$ belong to the kernel of $\varphi$.

To prove that they actually generate the kernel let us consider the image of the Macdonald polynomials $P_{\lambda}(z, q, t)$ with $\lambda \in H_{n, m}$. From the formula (22) it follows that the leading term in lexicographic order of $\varphi\left(P_{\lambda}(z, q, t)\right)$ has a form

$$
(-1)^{|\mu|} \frac{H(\mu, q, t)}{H\left(\mu^{\prime}, t, q\right)} x_{1}^{\lambda_{1}} \ldots x_{n}^{\lambda_{n}} y_{1}{ }^{\mu_{1}^{\prime}} \ldots y_{m}{ }^{\mu_{m}^{\prime}}
$$

where $\mu=\left(\lambda_{n+1}, \lambda_{n+2}, \ldots\right)$. From the definition $\varphi\left(P_{\lambda}(z, q, t)\right) \in \Lambda_{n, m, q, t}$. It is clear that all these polynomials corresponding to the diagrams contained in the fat hook are linearly independent. The Theorem is proved.

Note that we have also shown that for the generic $q, t$ the restriction of the Macdonald polynomials on the subvariety $\Delta_{n, m, q, t} \subset \mathcal{M}$

$$
S P_{\lambda}(x, y, q, t)=\varphi\left(P_{\lambda}(z, q, t)\right), \quad \lambda \in H_{n, m}
$$

forms a basis in $\Lambda_{n, m, q, t}$. By analogy with the Jack polynomials case (see e.g. [2]) we call $S P_{\lambda}(x, y, q, t)$ the super Macdonald polynomials.

Corollary 5.7. Let $f \in \Lambda_{t}$ be a shifted symmetric function and $\mathcal{M}_{q, t}^{f}=$ $\chi(f) \in \mathcal{D}_{q, t}$ be the corresponding difference operator commuting with the 
$M R$ operator $\mathcal{M}_{q, t}$. Then for generic $q, t$ there exists a difference operator $M_{n, m, q, t}^{f}$ commuting with the deformed $M R$ operator (1) such that the following diagram is commutative

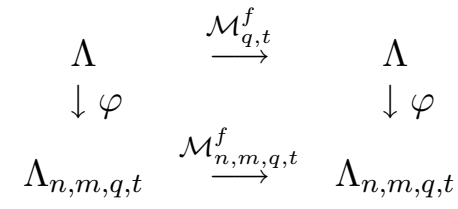

All the operators $M_{n, m, q, t}^{f}$ commute with each other and the super Macdonald polynomials (23) are their joint eigenfunctions.

Remark. For special $q, t$ of the form $q=t^{k}$ where $k$ is a nonnegative integer the homomorphism $\varphi$ can be passed through the finite dimension $N=n+m k: \varphi=\phi \circ \varphi_{N}$, where $\varphi_{N}: \Lambda \rightarrow \Lambda_{N}$ is the standard map (all $z_{i}$ except $N$ go to zero), and $\phi: \Lambda_{N} \rightarrow \Lambda_{n, m, q, t}$ is a homomorphism such that

$$
\phi\left(z_{i}\right)=x_{i}, i=1, \ldots, n, \quad \phi\left(z_{n+k l+j}\right)=t^{j-1} y_{l}, l=1, \ldots, m, j=1, \ldots, k .
$$

When $m=1$ the corresponding ideal (the kernel of $\phi$ ) was investigated by B. Feigin, Jimbo, Miwa and Mukhin in [9], who also described it in terms of Macdonald polynomials, but their description is much more complicated then in the generic parameters case. The case $k=2, m=2$ was studied in $[14]$.

We investigate the homomorphism: $f \rightarrow M_{n, m, q, t}^{f}$ in more detail in the next section, but here we finish with the following result mentioned at the beginning of this section.

Recall that the parameters $q, t$ are call special if $q^{a}=t^{b}$ for some nonnegative integers $a, b$ not equal to zero simultaneously.

Theorem 5.8. If the parameters $q, t$ are non-special then $\Lambda_{n, m, q, t}$ is generated by the deformed Newton sums $p_{r}(x, y, q, t)$.

The main idea is standard for this kind of result (see e.g. [1]). We show that the dimensions of the homogeneous components of the algebra $\Lambda_{n, m, q, t}$ and its subalgebra $\mathcal{N}_{n, m, q, t}$ generated by the deformed Newton sums are the same and thus these two algebras coincide. More precisely, we prove that these dimensions coincide with the number of the corresponding Young diagrams contained in the fat $(n, m)$-hook (see Fig. 1 above).

Lemma 5.9. If $q, t$ are not special then the dimension of the homogeneous component $\Lambda_{n, m ; q, t}$ of degree $N$ is less or equal to the number of partitions $\lambda$ of $N$ such that $\lambda_{n+1} \leq m$.

Proof. For a given partition $\nu$ consider the set of all different partitions $\hat{\nu}$, which one can get from $\nu$ by eliminating at most one part of it (or one row in the Young diagram representation). We have the following obvious formula

$$
m_{\nu}\left(x_{1}, x_{2}, \ldots, x_{n}\right)=\sum_{\hat{\nu} \cup(a)=\nu} x_{1}^{a} m_{\hat{\nu}}\left(x_{2}, \ldots, x_{n}\right)
$$


where $(a)$ denote the row of length $a$. Any element $f \in \Lambda_{n, m, q, t}$ can be written in the form

$$
f=\sum_{\lambda, \mu} c(\lambda, \mu) m_{\lambda}\left(x_{1}, x_{2}, \ldots, x_{n}\right) m_{\mu}\left(y_{1}, y_{2}, \ldots, y_{n}\right)
$$

Then from (11) and (24) we have the linear system

$$
\sum_{a+b=p}\left(q^{a}-t^{b}\right) c(\lambda \cup(a), \mu \cup(b))=0,
$$

where $a, b, p$ nonnegative integers and $\lambda, \mu$ are partitions such that $\lambda_{n}=$ $0, \mu_{m}=0$.

Consider the set $X_{N}(n, m)$ of pairs $\lambda, \mu$ of partitions such that $|\lambda|+|\mu|=$ $N, \lambda_{n+1}=0, \mu_{m+1}=0$. Then we have disjoint union

$$
X_{N}(n, m)=D_{N}(n, m) \cup R_{N}(n, m),
$$

where $D_{N}(n, m)$ is the subset of pairs $(\lambda, \mu)$ of partitions such that $\lambda_{n+1} \geq$ $\mu_{1}^{\prime}$. The pairs from $R_{N}(n, m)$ will be called irregular. Note that the corresponding Young diagrams $\lambda \cup \mu^{\prime}$, where $\mu^{\prime}$ corresponds to the transposed Young diagram, are precisely those which are contained in the fat $(n, m)$ hook. We would like to show that one can express $c(\lambda, \mu)$ for all others partitions as a linear combinations of those from $D_{N}(n, m)$.

Consider any $(\lambda, \mu) \in R_{N}(n, m): \lambda=\left(\lambda_{1}, \ldots, \lambda_{n}\right), \mu=\left(\mu_{1}, \ldots, \mu_{s}\right)$. Define

$$
k=\sharp\left\{i \mid \lambda_{i}<\mu_{1}^{\prime}\right\}
$$

and

$$
\mu(q)=\mu_{s-q+1}+\cdots+\mu_{s}
$$

for any integer $0<q \leq s$. Introduce the following partial order on $R_{N}(n, m)$ : $(\lambda, \mu) \prec(\tilde{\lambda}, \tilde{\mu})$ if and only if $k<\tilde{k}$ or one of the following conditions is fulfilled:

$k=\tilde{k}$ and for $q=\min \left\{\lambda_{n}, \tilde{\lambda}_{n}\right\}, \mu(q+1)<\tilde{\mu}(q+1)$

$k=\tilde{k}$ and for $q=\min \left\{\lambda_{n}, \tilde{\lambda}_{n}\right\}, \mu(q+1)=\tilde{\mu}(q+1)$ and $\lambda_{n}<\tilde{\lambda}_{n}$. We prove the lemma by induction in $N(x)=\sharp\left\{y \prec x \mid y \in R_{N}(n, m)\right\}$. Let $(\lambda, \mu) \in R_{N}(n, m)$. Consider equation (25) where $\hat{\lambda}=\left(\lambda_{1}, \ldots, \lambda_{n-1}\right)$ and $\hat{\mu}=\left(\mu_{1}, \ldots, \mu_{s-\lambda_{n}-1}, \mu_{s-\lambda_{n}+1}, \ldots, \mu_{s}\right)$ and $p=\lambda_{n}+\mu_{s-\lambda_{n}}$. It is easy to see that the equation contains $c(\lambda, \mu)$ with a non-zero coefficient and $(\lambda, \mu)$ is maximal among all irregular pairs in this equation.

Now let us prove the Theorem. To show that $\mathcal{N}_{n, m, q, t}=\Lambda_{n, m, q, t}$ it is enough to prove that the dimension of the homogeneous component of degree $N$ of $\mathcal{N}_{n, m, q, t}$ is not less than $D_{N}(n, m)$. To produce enough independent polynomials consider the super Macdonald polynomials (23)

$$
S P_{\lambda}(x, y, q, t)=\varphi\left(P_{\lambda}(z, q, t)\right), \quad \lambda \in H_{n, m} .
$$


From the formula $(22)$ it follows that the leading term of $\left.S P_{\lambda}(x, y, q, t)\right)$ in lexicographic order has a form

$$
x_{1}^{\lambda_{1}} \ldots x_{n}^{\lambda_{n}} y_{1}^{<\lambda_{1}^{\prime}-n>} \ldots y_{m}^{<\lambda_{m}^{\prime}-n>},
$$

where $\lambda^{\prime}$ is the partition conjugate to $\lambda$ and $\langle x\rangle=\frac{x+|x|}{2}=\max (0, x)$. From the definition $\varphi\left(P_{\lambda}(z, q, t)\right) \in \mathcal{N}_{n, m, q, t}$. It is clear that all these polynomials corresponding to the diagrams contained in the fat hook are linearly independent. This completes the proof of Theorem 5.8.

\section{Shifted super Macdonald polynomials and Harish-Chandra HOMOMORPHISM}

Let again $P_{n, m}=\mathbb{C}\left[x_{1}, \ldots, x_{n}, y_{1}, \ldots, y_{m}\right]$ be polynomial algebra in $n+m$ independent variables. The following algebra $\Lambda_{n, m, q, t}^{\natural}$ can be considered as a shifted version of the algebra $\Lambda_{n, m, q, t}$. It consists of the polynomials $p\left(x_{1}, \ldots, x_{n}, y_{1}, \ldots, y_{m}\right)$, which are symmetric in $x_{1}, x_{2} t, \ldots, x_{n} t^{n-1}$ and $y_{1}, y_{2} q \ldots, y_{m} q^{m-1}$ separately and satisfy the conditions

$$
T_{q, x_{i}}(f)=T_{t, y_{j}}(f)
$$

on each hyperplane $x_{i} t^{i-1}-y_{j} q^{j-1}=0$ for $i=1, \ldots, n$ and $j=1, \ldots, m$.

Now we are going to define the homomorphism $\varphi^{\natural}$ which is a shifted version of the homomorphism $\varphi$ from the previous section.

Recall that $H_{n, m}$ denote the set of partitions $\lambda$ whose diagrams are contained in the fat $(n, m)$-hook. Consider the following $F: H_{n, m} \longrightarrow \mathbb{C}^{n+m}$ : $F(\lambda)=\left(p_{1}, \ldots, p_{n}, q_{1}, \ldots, q_{m}\right)$, where

$$
p_{i}=q^{\lambda_{i}}, \quad q_{j}=t^{\mu_{j}^{\prime}} t^{n},
$$

and $\mu=\left(\lambda_{n+1}, \lambda_{n+2}, \ldots\right)$. The image $F\left(H_{n, m}\right)$ is dense in $\mathbb{C}^{n+m}$ with respect to the Zariski topology. Indeed, this is clearly true already for the subset consisting of the corresponding partitions with $\lambda_{n} \geq m$. The homomorphism

$$
\varphi^{\natural}: \Lambda_{t} \longrightarrow \mathbf{C}\left[x_{1}, \ldots, x_{n}, y_{1}, \ldots, y_{m}\right]
$$

is defined by the relation

$$
\varphi^{\natural}(f)\left(p_{1}, \ldots, p_{n}, q_{1}, \ldots, q_{m}\right)=f\left(q^{\lambda}\right),
$$

where $\left(p_{1}, \ldots, p_{n}, q_{1}, \ldots, q_{m}\right) \in F\left(H_{n, m}\right)$ and $\lambda=F^{-1}\left(p_{1}, \ldots, p_{n}, q_{1}, \ldots, q_{m}\right)$. In other words, we consider the shifted symmetric function $f$ as a function on the partitions from the fat hook and re-write it in the new coordinates. The fact that as a result we will have a polynomial is not obvious.

Lemma 6.1. The image $\varphi^{\natural}(f)$ of a shifted symmetric function $f \in \Lambda_{t}$ is a polynomial. For the shifted power sums $p_{k}^{*}(z, t)$ it can be given by the following explicit formula:

$$
\varphi^{\natural}\left(p_{k}^{*}(z, t)\right)=\sum_{i=1}^{n}\left(x_{i}^{r}-1\right) t^{r(i-1)}+\frac{1-q^{r}}{1-t^{r}} \sum_{j=1}^{m}\left(y_{j}^{r}-t^{r n}\right) q^{r(j-1)} .
$$


Proof. Assume that $z_{i}=q^{\lambda_{i}}$, where $\lambda \in H_{n, m}$. Then we have

$\varphi^{\natural}\left(p_{k}^{*}(z, t)\right)=\sum_{i \geq 1}\left(q^{r \lambda_{i}}-1\right) t^{i-1}=\sum_{i=1}^{n}\left(q^{r \lambda_{i}}-1\right) t^{r(i-1)}+t^{r n} \sum_{i \geq 1}\left(q^{r \lambda_{n+i}}-1\right) t^{r(i-1)}$.

Now using (8) we have

$$
\sum_{i \geq 1}\left(q^{r \lambda_{n+i}}-1\right) t^{r(i-1)}=\frac{1-q^{r}}{1-t^{r}} \sum_{j=1}^{m}\left(t^{r \mu_{j}^{\prime}}-1\right) q^{r(j-1)}
$$

which proves the formula (27). Since the shifted sums generate $\Lambda_{t}$ this implies the first part of the lemma as well.

Theorem 6.2. If the parameters $q, t$ are generic then the image of the homomorphism $\varphi^{\natural}$ coincides with the algebra $\Lambda_{n, m, q, t}^{\natural}$ and the kernel of $\varphi^{\natural}$ is spanned by the shifted Macdonald polynomials $P_{\lambda}^{*}(z, q, t)$ corresponding to the Young diagrams which are not contained in the fat $(n, m)$-hook.

Proof. The first claim follows from Lemma 6.1 and Theorem 5.8. To prove the statement about the kernel consider a shifted Macdonald polynomial $P_{\lambda}^{*}(z, q, t)$ with $\lambda \in \bar{H}_{n, m}$. Let $\mu$ be a partition whose diagram is contained in the fat $(n, m)$-hook. Since this implies that the diagram of $\lambda$ is not a subset of the one of $\mu$ according to the Extra Vanishing Property of shifted Macdonald polynomials (see Section 3 ) we have $P_{\lambda}^{*}\left(q^{\mu}, q, t\right)=0$. Thus we have shown that $P_{\lambda}^{*}(z, q, t)$ with $\lambda \in \bar{H}_{n, m}$ belong to the kernel of $\varphi^{\natural}$. To show that they generate the kernel one should note that

$\varphi^{\natural}\left(P_{\lambda}^{*}(z, q, t)\right)=\varphi\left(P_{\lambda}(z, q, t)\right)\left(x_{1}, x_{2} t, \ldots, x_{n} t^{n-1}, y_{1}, y_{2} q \ldots, y_{m} q^{m-1}\right)+\ldots$,

where dots mean the terms of degree less then $|\lambda|$. From theorem 5.6 it follows that $\varphi^{\natural}\left(P_{\lambda}^{*}(z, q, t)\right)$ with $\lambda \in H_{n, m}$ are linearly independent. The theorem is proved.

Corollary 6.3. For generic $q, t$ the functions

$$
S P_{\lambda}^{*}(x, y, q, t)=\varphi^{\natural}\left(P_{\lambda}^{*}(z, q, t)\right)
$$

with $\lambda \in H_{n, m}$ form a basis in $\Lambda_{n, m, q, t}^{\natural}$.

We will call the polynomials $S P_{\lambda}^{*}(x, y, q, t)$ the shifted super Macdonald polynomials. We are going to show that to any such polynomial corresponds a quantum integral of the deformed MR system.

Let us consider the algebra of difference operators in $n+m$ variables with rational coefficients belonging to $\mathbb{C}\left[x_{1}, \ldots, x_{n}, y_{1}, \ldots, y_{m},\left(x_{i}-x_{j}\right)^{-1},\left(x_{i}-\right.\right.$ $\left.\left.\left.y_{l}\right)^{-1},\left(y_{k}-x_{l}\right)^{-1}\right)\right], \quad 1 \leq i<j \leq n, 1 \leq l<k \leq m$. We denote it as $\Delta(n, m)$. 
Theorem 6.4. For generic values of $q, t$ there exists a unique monomorphism $\psi: \Lambda_{n, m, q, t}^{\natural} \rightarrow \Delta(n, m)$ such that the following diagram is commutative

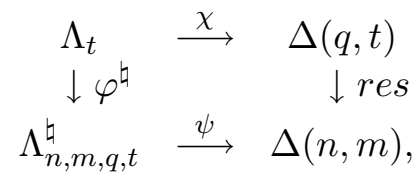

where $\chi$ is the inverse Harish-Chandra homomorphism and res is the operation of restriction onto $\Delta(n, m, q, t)$ described by Corollary 5.7.

Indeed let $f$ be a shifted symmetric function from $\Lambda_{t}, \mathcal{M}_{q, t}^{f}$ and $\mathcal{M}_{n, m, q, t}^{f}=$ $\operatorname{res}\left(\mathcal{M}_{q, t}^{f}\right)$ be the same as in Corollary 5.7. We know that if $P_{\lambda}(z, q, t)$ is a Macdonald symmetric function then

$$
\mathcal{M}_{n, m, q, t}^{f} \varphi\left(P_{\lambda}(z, q, t)\right)=f\left(q^{\lambda}\right) \varphi\left(P_{\lambda}(z, q, t)\right) .
$$

Therefore according to Theorem $5.6 \mathcal{M}_{n, m, q, t}^{f} \equiv 0$ if and only if $f\left(q^{\lambda}\right)=0$ for any $\lambda$ with the diagram contained in the fat $(n, m)$-hook. Now from Theorem 6.2 it follows that $\operatorname{Ker}(r e s \circ \chi)=\operatorname{Ker} \varphi^{\natural}$.

\section{Combinatorial Formulas}

In this section we give some combinatorial formulas for the super Macdonald polynomials and shifted super Macdonald polynomials generalising the results by Okounkov [8]. Let us recall his results.

A tableau $T$ on $\lambda$ is called a reverse tableau if its entries decrease strictly downwards in each column and weakly rightwards in each row. By $T(s)$ we denote the entry in the box $s \in \lambda$. The following combinatorial formula for the shifted Macdonald polynomial was proven by Okounkov in [8]:

$$
P_{\lambda}^{*}\left(x_{1}, \ldots, x_{N}, q, t\right)=\sum_{T} \psi_{T}(q, t) \prod_{s \in \lambda}\left(x_{T(s)}-q^{a^{\prime}(s)} t^{l^{\prime}(s)}\right) t^{T(s)-1}
$$

where $a^{\prime}(s)$ and $l^{\prime}(s)$ are defined for a box $s=(i, j)$ as

$$
a^{\prime}(s)=j-1, \quad l^{\prime}(s)=i-1 .
$$

Here the sum is taken over all reverse tableaux on $\lambda$ with entries in $\{1, \ldots, N\}$ and $\psi_{T}(q, t)$ is the same weight as in the combinatorial formulas for the ordinary Macdonald polynomials (see [4], VI, (7.13')) interpreted in terms of reverse tableau:

$$
\begin{aligned}
P_{\lambda}\left(x_{1}, \ldots, x_{N}, q, t\right) & =\sum_{T} \psi_{T}(q, t) \prod_{s \in \lambda} x_{T(s)} . \\
P_{\lambda / \mu}\left(x_{1}, \ldots, x_{N}, q, t\right) & =\sum_{T} \psi_{T}(q, t) \prod_{s \in \lambda / \mu} x_{T(s)} .
\end{aligned}
$$

In the last formula the sum is taken over all reverse tableaux of shape $\lambda / \mu$ with entries in $\{1, \ldots, N\}$. 
Let us consider now a reverse bitableau $T$ of type $(n, m)$ and shape $\lambda$. We can view $T$ as a filling of a Young diagram $\lambda$ by symbols

$$
1<2<\cdots<n<1^{\prime}<2^{\prime}<\cdots<m^{\prime}
$$

with entries decreasing weakly downwards in each column and rightwards in each row; additionally entries $1,2 \ldots, n$ decrease strictly downwards in each column and entries $1^{\prime}, 2^{\prime} \ldots, m^{\prime}$ decrease strictly rightwards in each row. Here is an example of a reverse bitableau of type $(3,2)$ :

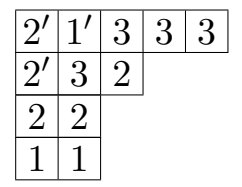

Let $T_{1}$ be a subtableau of $T$ containing all symbols $1^{\prime}, 2^{\prime} \ldots, m^{\prime}, \mu$ is its shape and $T_{0}=T-T_{1}$. Note that the conjugate tableau $T_{1}^{\prime}$ is the usual reverse tableau (if we ignore prime symbols) and $T_{0}$ is the usual reverse skew tableau of shape $\lambda / \mu$. In the rest of this section $x_{j^{\prime}}$ will be denoted as $y_{j}$.

Theorem 7.1. For generic values of parameters $q, t$ the super Macdonald polynomials can be written as

$$
S P_{\lambda}\left(x_{1}, x_{2}, \ldots, x_{n}, y_{1}, y_{2}, \ldots, y_{m}, q, t\right)=\sum_{T} \psi_{T}(q, t) \prod_{s \in \lambda} x_{T(s)},
$$

where the sum is taken over all reverse bitableaux $T$ of type $(n, m)$ and shape $\lambda$ and

$$
\psi_{T}(q, t)=(-1)^{|\mu|} \psi_{T_{1}^{\prime}}(t, q) \psi_{T_{0}}(q, t) \frac{H(\mu, q, t)}{H\left(\mu^{\prime}, t, q\right)}
$$

with $H(\mu, q, t)$ defined by (5).

The proof follows directly from the formulas $(22),(29),(30)$.

We are going to present now a combinatorial formula for the shifted super Macdonald polynomial.

Theorem 7.2. The following formula holds:

$$
S P_{\lambda}^{*}=\sum_{T} \psi_{T}(q, t) \prod_{s \in \lambda}\left(x_{T(s)}-q^{a^{\prime}(s)} t^{l^{\prime}(s)}\right)(q, t ; s)^{T(s)-1}
$$

where the sum is taken over the same set of reverse bitableaux as in previous theorem, $(q, t ; s)=q$ if $s \in T_{1}$ and $(q, t ; s)=t$ if $s \in T_{0}$.

Proof. Let us consider the skew diagram $\lambda / \mu$ in the formula (29) and define

$$
P_{\lambda / \mu}^{*}(x, q, t)=\sum_{T} \psi_{T}(q, t) \prod_{s \in \lambda / \mu}\left(x_{T(s)}-q^{a^{\prime}(s)} t^{l^{\prime}(s)}\right) t^{T(s)-1} .
$$

Okounkov in [8] proved the following formula

$$
P_{\lambda}^{*}\left(z_{1}, z_{2} \ldots, q, t\right)=\sum_{\mu \prec \lambda} \psi_{\lambda / \mu}(q, t) \prod_{s \in \lambda / \mu}\left(z_{1}-q^{a^{\prime}(s)} t^{l^{\prime}(s)}\right) t^{|\mu|} P_{\mu}^{*}\left(z_{2}, z_{3} \ldots, q, t\right),
$$


where $\mu \prec \lambda$ means $\lambda_{i+1} \leq \mu_{i} \leq \lambda_{i}$ and $\psi_{\lambda / \mu}(q, t)$ is the same coefficient as for the ordinary Macdonald polynomials [4]

$$
P_{\lambda}\left(z_{1}, z_{2} \ldots, q, t\right)=\sum_{\mu \prec \lambda} \psi_{\lambda / \mu}(q, t) z_{1}^{|\lambda / \mu|} P_{\mu}\left(z_{2}, z_{3} \ldots, q, t\right) .
$$

Strictly speaking Okounkov proved this for finitely many variables. To make sense of formula (33) in infinite dimension we embed the algebra $\Lambda_{t}$ to $\mathbb{C}\left[z_{1}\right] \otimes \Lambda_{t}$ by sending $p_{k}^{*}$ to $z_{1}^{k}-1+t^{k} p_{k}^{*}$.

Applying Okounkov's formula $n$ times we get

$$
P_{\lambda}^{*}\left(z_{1}, z_{2} \ldots, q, t\right)=\sum_{\mu \subset \lambda} P_{\lambda / \mu}^{*}\left(z_{1}, z_{2}, \ldots, z_{n}, q, t\right) t^{n|\mu|} P_{\mu}^{*}\left(z_{n+1}, z_{n+2} \ldots, q, t\right),
$$

which implies

$$
\varphi^{\natural}\left(P_{\lambda}^{*}\left(z_{1}, z_{2} \ldots, q, t\right)\right)=\sum_{\mu \subset \lambda} P_{\lambda / \mu}^{*}\left(x_{1}, x_{2}, \ldots, x_{n}, q, t\right) t^{n|\mu|} \omega_{q, t}^{*}\left(P_{\mu}^{*}\left(z_{n+1}, z_{n+2} \ldots, q, t\right)\right) .
$$

Now using the duality (6) we have

$$
\varphi^{\natural}\left(P_{\lambda}^{*}\left(z_{1}, z_{2} \ldots, q, t\right)\right)=\sum_{\mu \subset \lambda} P_{\lambda / \mu}^{*}\left(x_{1}, x_{2}, \ldots, x_{n}, q, t\right) \frac{H(\mu, q, t)}{H\left(\mu^{\prime}, t, q\right)} P_{\mu^{\prime}}^{*}\left(y_{1}, y_{2} \ldots, y_{m}, t, q\right) .
$$

But according to formula (28)

$$
\begin{gathered}
P_{\mu^{\prime}}^{*}\left(y_{1}, y_{2} \ldots, y_{m}, t, q\right)=\sum_{T_{1}^{\prime}} \psi_{T_{1}^{\prime}}(t, q) \prod_{s^{\prime} \in \mu^{\prime}}\left(x_{T_{1}^{\prime}\left(s^{\prime}\right)}-t^{a^{\prime}\left(s^{\prime}\right)} q^{l^{\prime}\left(s^{\prime}\right)}\right) q^{T_{1}^{\prime}\left(s^{\prime}\right)-1} \\
=\sum_{T_{1}} \psi_{T_{1}^{\prime}}(t, q) \prod_{s \in \mu}\left(x_{T_{1}}(s)-q^{a^{\prime}(s)} t^{l^{\prime}(s)}\right) q^{T_{1}(s)-1}
\end{gathered}
$$

Therefore

$$
\begin{gathered}
\varphi^{\natural}\left(P_{\lambda}^{*}\left(z_{1}, z_{2} \ldots, q, t\right)\right)=\sum_{T} t^{|\mu|} \frac{H(\mu, q, t)}{H\left(\mu^{\prime}, t, q\right)} \psi_{T}(q, t) \prod_{s \in \lambda / \mu}\left(x_{T}(s)-q^{a^{\prime}(s)} t^{l^{\prime}(s)}\right) t^{T(s)-1} \\
\psi_{T_{1}^{\prime}}(t, q) \prod_{s \in \mu}\left(x_{T_{1}}(s)-q^{a^{\prime}(s)} t^{l^{\prime}(s)}\right) q^{T_{1}(s)-1}
\end{gathered}
$$

and the theorem is proved.

\section{Concluding Remarks}

Haglund, Haiman and Loehr [16] recently proved a remarkable new combinatorial formula for Macdonald polynomials, previously proposed by Haglund. These formulas are much more effective than the original Macdonald's formulas (29) and provide a new direct way to prove the main properties of Macdonald polynomials. A natural problem is to find an analogue of Haglund's formula for the super Macdonald polynomials. We would like to mention here recent work by Ram and Yip [17], where Haglund's formula was generalised for other Lie algebras. 
Another interesting open problem is to find generalisations of our results for the deformed analogues of the Macdonald operators related to other Lie superalgebras, in particular for the deformed Koornwinder operators (see the rational limit of them in [18]).

\section{ACKNowledgments}

We are grateful to A. Okounkov for useful and stimulating discussions. Special thanks go to the anonymous referee for an excellent job, which helped us to improve the paper.

This work has been partially supported by the EPSRC (grant EP/E004008/1), European Union through the FP6 Marie Curie RTN ENIGMA (Contract number MRTN-CT-2004-5652) and ESF programme MISGAM.

\section{REFERENCES}

[1] A.N. Sergeev, A.P. Veselov Deformed quantum Calogero-Moser Problems and Lie superalgebras. Comm. Math. Phys. 245 (2004), no. 2, 249-278.

[2] A.N. Sergeev, A.P. Veselov Generalised discriminants, deformed Calogero-MoserSutherland operators and super-Jack polynomials. Adv. Math. 192 (2005), no. 2, 341375 .

[3] S.N.M. Ruijsenaars Complete integrability of relativistic Calogero-Moser systems and elliptic function identities. Comm. Math. Phys. 110 (1987), no. 2, 191-213.

[4] I. Macdonald Symmetric functions and Hall polynomials 2nd edition, Oxford Univ. Press, 1995.

[5] F. Knop, S. Sahi, Difference equations and symmetric polynomials defined by their zeros. Internat. Math. Res. Notes 10, 1996, 437-486.

[6] F. Knop Symmetric and non-symmetric quantum Capelli polynomials. Comment. Math. Helv. 72 (1997), no. 1, 84-100.

[7] S. Sahi Interpolation, integrality, and a generalization of Macdonald's polynomials. Internat. Math. Res. Notices (1996), no. 10, 457-471.

[8] A. Okounkov (Shifted) Macdonald polynomials : q-integral representation and combinatorial formula. Compositio Math. 112, 1998, no.2, 147-182.

[9] B. Feigin, M. Jimbo, T. Miwa, E., Mukhin.Symmetric polynomials vanishing on the shifted diagonals and Macdonald polynomials. Int. Math. Res. Not. 2003, no. 18, 1015-1034.

[10] O.A. Chalykh Macdonald polynomials and algebraic integrability. Adv. Math. 166 (2002), no. 2, 193-259.

[11] A.P. Veselov, M.V. Feigin, O.A. Chalykh New integrable deformations of quantum Calogero - Moser problem. Russian Math. Surveys 51, no.3, 1996, 185-186.

[12] I. Cherednik, Double affine Hecke algebras and Macdonald's conjectures, Ann. of Math. (2) 141 (1995), no. 1, 191-216.

[13] A.N. Kirillov, M. Noumi Affine Hecke algebras and raising operators for Macdonald polynomials. Duke Math. J. 93 (1998), no. 1, 1-39.

[14] M. Kasatani, T. Miwa, A.N. Sergeev, A.P. Veselov Coincident root loci and Jack and Macdonald polynomials for special values of the parameters. Jack, Hall-Littlewood and Macdonald polynomials, 207-225, Contemp. Math., 417, Amer. Math. Soc., Providence, RI, 2006.

[15] M. Atiyah, I.G. Macdonald Introduction to Commutative Algebra. Addison-Wesley, 1969.

[16] J. Haglund, M. Haiman, N. Loehr A combinatorial formula for Macdonald polynomials. J. Amer. Math. Soc. 18 (2005), no. 3, 735-761. 
[17] A. Ram, M. Yip A combinatorial formula for Macdonald polynomials. arXiv:0803.1146.

[18] A.N. Sergeev, A.P. Veselov BC-infinity Calogero-Moser operator and super Jacobi polynomials. arXiv:0807.3858.

Department of Mathematical Sciences, Loughborough University, LoughBorough LE11 3TU, UK and Steklov Institute of Mathematics, Fontanka 27, St. Petersburg, 191023, Russia

E-mail address: A.N.Sergeev@lboro.ac.uk

Department of Mathematical Sciences, Loughborough University, LoughBorough LE11 3TU, UK and Landau Institute for Theoretical Physics, Moscow, RUSSIA

E-mail address: A.P.Veselov@lboro.ac.uk 